\title{
Myocardial Ischaemia Due to Ruptured Left Sinus of Valsalva - A Case Report
}

\author{
MASOOM SIRAJ, MD. SHARIF HASAN \\ Department of Cardiac Surgery, Ibrahim Cardiac Hospital and Research Institute, Shahbag, Dhaka \\ Address for correspondence: Masoom Siraj, Department of cardiac Surgery, Ibrahim Cardiac Hospital and Research Institute \\ Shahbagh, Dhaka
}

\begin{abstract}
Here we present a case involving a middle aged male patient suffering from ruptured left Sinus Of Valsalva (SOV) and attendant complications. The rarity of the condition presents a clinical dilemma in the diagnosis and treatment of the same. Occurrences are so rare that not much comprehensive guideline is available for the medical team to proceed with treatment. We describe how we arrived at the diagnosis by correlating the various investigations done and also the surgical correction done resulting in a satisfactory outcome.
\end{abstract}

Keywords: Sinus of Valsalva, Aneurysm, Ischaemia

\section{Introduction:}

Sinuses of Valsalva (SOV) are three small outpouchings at the root of the aorta. Each takes its name from its relation to the origin of a coronary artery. Therefore we have a Left SOV, a Right SOV and a Non-coronary SOV. Rupture of a sinus of valsalva is a rare condition. Its manifestations are protean with an asymptomatic murmur in one end to as acute, cardiogenic shock leading to death at the other extreme. This great variation is influenced by the SOV involved and the cavity it ruptures into. Diagnosing the condition is a challenge not only because of the rarity of the condition but also because the wide variety of symptoms given. How to proceed with a surgical correction is also not clearly outlined because of the same reason. Here we present how we arrived at a diagnosis by correlating the symptoms and the multitude of investigations done. We also describe our surgical correction resulting in a satisfactory outcome.

\section{Case Summary:}

In February 2010 a forty five year old gentleman got admitted to our hospital with a three months long history of palpitation and breathlessness which had become severe in the last three days. On further questioning he also revealed suffering from episodes of unexplained fever for a few years. For this he attended the local doctor who prescribed him antibiotics. As he always responded to the treatment he did not go for further investigations and accepted the inconvenience as a fact of life. However this time he could sense things were different. Difficulty in breathing was the main symptom and was unusually severe compared to previous episodes. Palpitation was mild and fever was not a feature this time.

On admission he had a blood pressure of 110/60 mm of $\mathrm{Hg}$, heart rate of 92 per minute and regular. Auscultation revealed a systolic cardiac murmur and a clear chest. Respiratory difficulty had subsided by the time he came to the hospital.

Laboratory test results were all within normal limits. ECG was normal. Chest X-Ray showed mild cardiomegaly and small bilateral pleural effusion.

A Trans Thoracic Echo Cardiography (TTE) done showed the following: All the valves had normal morphological characteristics. There was severe eccentric aortic regurgitation (AR) and mild mitral regurgitation (MR), aneurysmal dilatation of the base of the interventricular septum and part of the root of the aorta. Good ejection fraction (EF\%) calculated at $60 \%$.

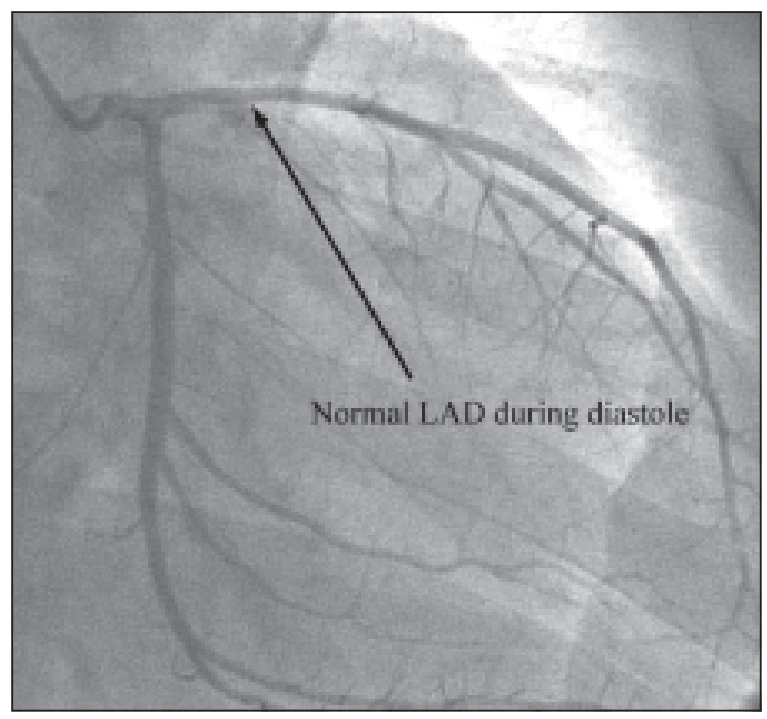

Fig.-1: Normal LAD during diastole. 
Next a coronary angiogram (CAG) was done. Epicardial coronary vessels were normal during diastole (Fig-1) but proximal part of left anterior descending (LAD) was being stretched and compressed during systole (Fig-2). A root aortogram clearly showed dye entering an aneurysmal sac from the root of the aorta (Fig-3) and from there making its way into the left ventricle (Fig-4).

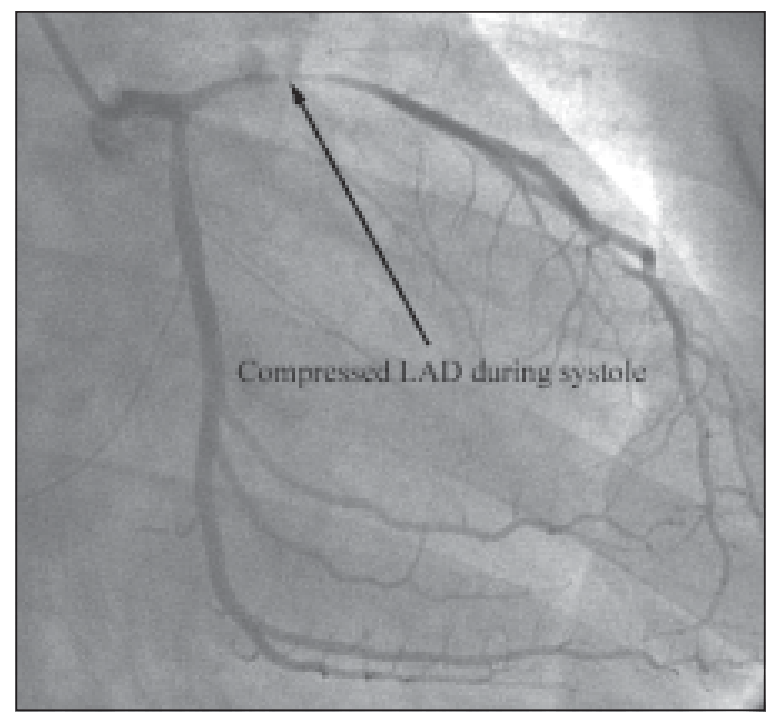

Fig.-2: Compressed LAD during systole.

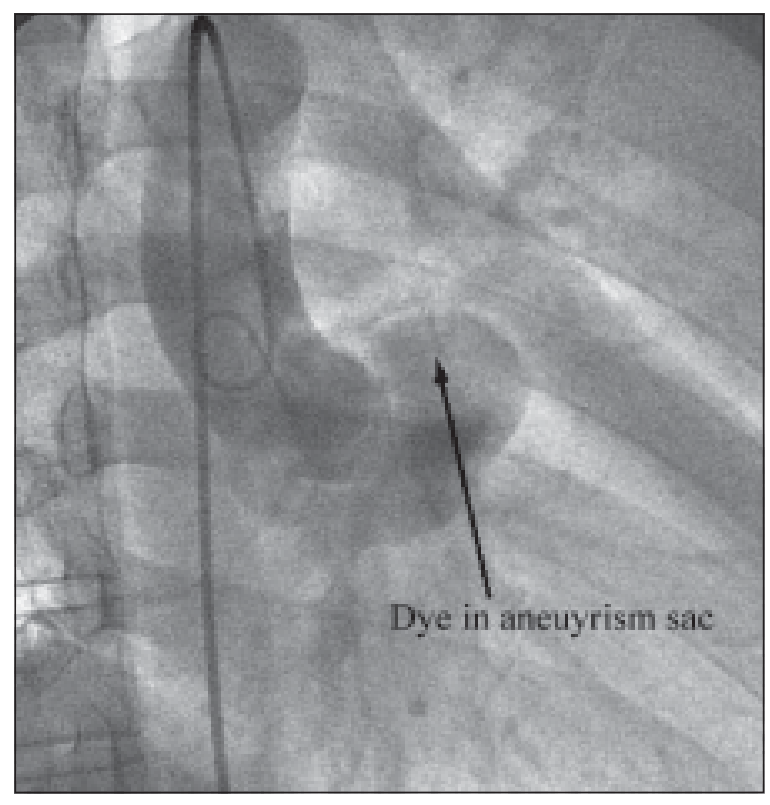

Fig.-3: Dye in aneurysm sac.

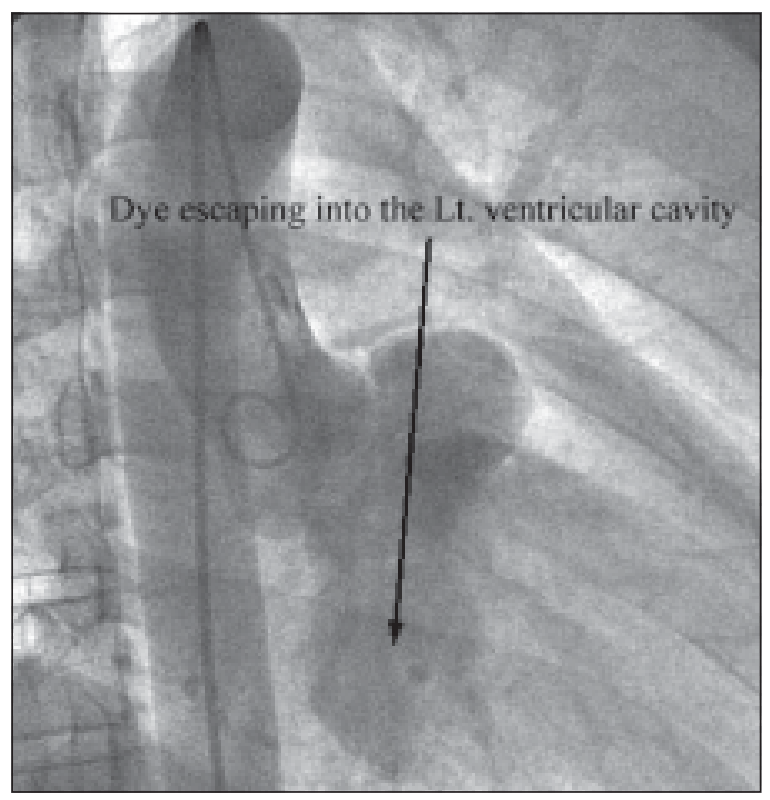

Fig.-4: Dye escaping into Lt. ventricular cavity.

A CT angiogram was done. This showed an outpouching in the region of the left coronary sinus just below the root of the aorta. This suspect aneurysm was compressing the proximal LAD.

Now a trans-esophageal echocardiography (TEE) was done. This showed an aneurysm arising from the left Sinus of Valsalva dissecting into the interventricular septum and opening into the left ventricle.

While we were investigating the patient he again got his "usual” febrile episode. He was put on antibiotics. Blood investigations did not reveal anything.

The case was discussed in the combined cardiologycardiac surgery meeting. Conclusion was this was a very rare case of aneurysm of the left sinus of Valsalva which had dissected into the interventricular septum and the recent exacerbation of symptoms has been due to rupture into the left ventricle. A decision for immediate surgical closure of the aneurysm with aortic valve replacement if necessary was taken. Despite the urgency of the clinical situation the sad truth about the economic scenario of our country surfaced. He did not have the funds necessary to pay for his surgery. While his relatives and family took time to arrange the finances, another very rarely reported complication happened.

The patient complained of severe central chest discomfort with sweating. He was hypotensive with significant STsegment changes in the ECG. He was immediately shifted to the coronary care unit for medical management. The 
diagnosis of myocardial infarction was further confirmed by significant elevation of Troponin - I. We agreed the ischaemic event was a result of the LAD being stretched and compressed by the left SOV aneurysm. The patient needed inotropioc support for haemodynamic stabilization. A repeat TTE was done. This now showed a drop in ejection fraction to $45 \%$ from the previous normal estimation of $60 \%$. The patient was again discussed with the cardiologists and it was decided to stabilize him medically and then take him to the operation theatre. We also hoped by this time the patient would generate the necessary funds which he did.

The heart was accessed via a median sternotomy. The patient was attached to the Heart-Lung machine with aortic arterial and bi-caval venous cannulae with snugging tapes on the ready. The patient was cooled down on bypass to 32 degree Celsius. Aorta was cross-clamped and aortotomy was made. The heart was arrested by direct cold osteal antegrade cardioplegia. The patient was further cooled down to 28 degree Celsius. Aortic valve was found to be normal. There was an opening in the left coronary sinus of Valsalva well clear of both the aortic valve and the left coronary osteum. A blunt metal probe was gently passed. It entered an aneurysmal sac on the epicardial side of the root of the aorta. The sac had also dissected into the interventricular septum. However the metal probe did not exit into the left ventricle and no force was applied on it. Now cold saline was injected into the opening. This made the aneurysmal sac. The left ventricular cavity was viewed through the aortic valve after retracting the leaflets. Though no exit opening could be seen, a jet of saline was seen jetting into the left ventricular cavity from somewhere outside the line of vision. After a quick discussion it was decided to close the opening of the aneurysm without disturbing anything else as that had the potential to create unwanted complications. The opening was closed with a native pericardial patch using interrupted pledget 5/0 Prolene sutures. Pledgets were placed within the aneurysm sac dissecting into the interventricular septum. Aortotomy was closed, patient was rewarmed and rest of the procedure went smoothly. Coming off bypass the aneurysmal sac at the root of the aorta was no longer bulging. Bypass time was 128 minutes and cross clamp time was 91 minutes.

The patient made a smooth recovery. He was extubated within two hours of arrival in the intensive care unit, was mobilised the next morning and was discharged on the $6^{\text {th }}$ post operative day. His complaints were gone and he felt better. A repeat TTE showed no aortic regurgitation this time, no paravalvular leak in any aneurysm or otherwise, no aneurysm sac below the root of the aorta. As of today the patient has not had any complications.

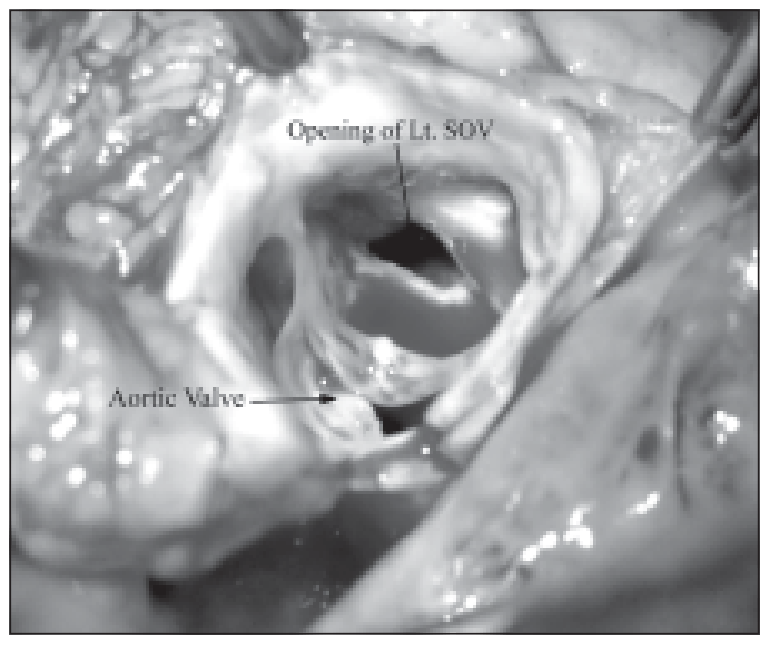

Fig.-5: Opening of left sinus of valsalva.

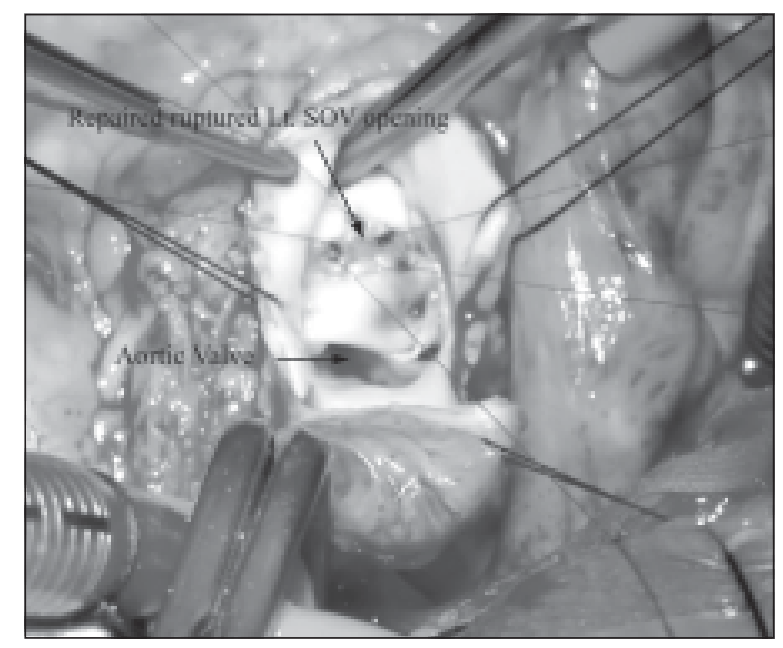

Fig.-6: Repair of ruptured s8inus of valsalva opening.

\section{Discussion}

A sinus of Valsalva aneurysm is an uncommon finding. It is found only in approximately less than $1 \%$ of patients undergoing cardiac surgical procedures. ${ }^{1}$ These are most often congenital in origin caused by a deficiency in the muscular and elastic tissue in the aortic wall behind the sinus of valsalva. ${ }^{2}$ Acquired causes of sinus of valsalva include infections (tuberculosis, tertiary syphilis, bacterial endocarditis); degenerative conditions (cystic medial necrosis, atherosclerosis) or trauma ${ }^{3,4}$ Males are more often affected with a ratio of 3:1 against females., 
Caucasians are less often affected compared to eastern population. ${ }^{6}$ The right coronary sinus in affected in more than $90 \%$ of cases, next common is the non- coronary sinus with only $1 \%$ cases involving the left coronary sinus ${ }^{7}$ .A sinus of valsalva burrows into the neighboring structures as it grows and notorious for remaining silent. However it may cause life threatening clinical conditions once it ruptures. Aneurysms involving the right sinus of Valsalva have a tendency to rupture into the right ventricle and those of the non-coronary sinus into the right atrium. ${ }^{1,6}$ The very rare aneurysm of the left sinus of valsalva may rupture into the pulmonary artery. ${ }^{8}$

Because of the rarity of the situation and also the absence of controlled trials, surgical management of an aneurysm of the left sinus of Valsalva is best guided by anecdoctal experience. We were faced with a situation where the anatomical involvement was the rarest subtype of a very rare pathological condition. Furthermore this exhibited two rare complications at the same time. Firstly left SOV had dissected into the interventricular septum and ruptured into the left ventricle. This has been reported before. Such a rupture may result in a spectrum of clinical scenario ranging from normal haemodynamics to a catastrophic heart failure. Our patient maintained his haemodynamic status. There are a few reported cases of a left SOV causing ischaemia. But none reported a case that a rupture had caused ischaemia. It is recommended for ruptured SOV aneurysms be surgically treated by controlling the point of entry and the point of exit. But we were not able to fulfill that recommendation as that could have created new complications. In our case we successfully took care of the situation by patch closure of the opening alone with a good surgical and clinical outcome.

Very few such cases have been published and we sincerely hope our experience will contribute towards helping other surgeons help their patients better.

\section{References:}

1. Takach TJ,Reul GJ,Duncan JM,Cooley DA,Livesay JJ,Ott DA,Frazier OH. Sinus of Valsalva sinus or fistula: management and outcome. Ann Thorac Surg 1999;68:1573-77.

2. Edwards JE,Burchell HB. The pathological anatomy of deficiencies between the aortic root and the heart, including aortic sinus aneurysms. Thorax 1957; 12: 125-39.

3. Ikenaga S,Minami Y,Itoh h,Suzuki K,Hamano K.Acceleration of aortic regurgitation due to localized aortic dissection; report of a case[in Japanese].Koyubu geka 2004; 57: 388-90.

4. Zhao G,Seng J,Yan B,Wei H,Qiao C,Zhao S, et al. Diagnosis and surgical treatment of ruptures aneurysm in sinus of valsalva. Chin Med J (Engl) 2003; 116: 1047-50.

5. .Friedman WF, Silverman N. In: Braunwald E, Zipes DP,Libby P, editors, Heart disease: a textbook of cardiovascular medicine. $6^{\text {th }}$ ed. Philadelphia: saunders; 2001. P 1540

6. Dong C, Wu QY, Tang Y. Ruptured sinus of valsalva aneurysm: a Beijing experience. Ann Thorac Surg 2002;74:1621-24

7. Guo DW, Cheng TO, Lin ML, Gu ZQ. Aneurysm of the sinus of valsalva:a roentgenologic study of 105 chinese patients. Am Heart j 1987;114:1169-77

8. Luckraz H, Naik M, Jenkins G, Youhana A. Repair of a sinus of valsalva that had ruptured into the pulmonary artery. J Thorac Cardiovasc surg 2004;127:1823-25. 